\title{
Radiomics in multiple sclerosis and neuromyelitis optica spectrum
}

\section{disorder}

\section{Abstract}

Objective: To develop and validate an individual radiomics nomogram for differential diagnosis between MS and NMOSD.

Methods: We retrospectively collected $67 \mathrm{MS}$ and 68 NMOSD with spinal cord lesions as a primary cohort, and prospectively recruited $28 \mathrm{MS}$ and 26 NMOSD patients as a validation cohort. Radiomic features were extracted from the spinal cord lesions. A prediction model for differentiating MS and NMOSD was built by combining the radiomic features with several clinical and routine MRI measurements. The performance of the model was assessed with respect to its calibration plot and clinical discrimination in the primary and validation cohorts.

Results: Nine radiomics features extracted from an initial set of 485 , predominantly reflecting lesion heterogeneity, combined with lesion length, patient sex, and EDSS, were selected to build the model for differentiating MS and NMOSD. The areas under the ROC curves (AUC) for differentiating the two diseases were 0.8808 and 0.7115 , for the primary and validation cohort, respectively. This model demonstrated good calibration (C-index was 0.906 and 0.802 in primary and validation cohort). Conclusions: A validated nomogram that incorporates the radiomics signature of spinal cord lesions, as well as cord lesion length, sex, and EDSS score, can usefully differentiate MS and NMOSD. 
Key words: multiple sclerosis; neuromyelitis optica spectrum disorder; radiomics; nomogram; MRI

\section{Key points:}

1: Radiomic features of spinal cord lesions in MS and NMOSD were different.

2: Radiomics signature can capture pathological alterations and help differentiate MS and NMOSD. 


\section{Introduction}

Multiple sclerosis (MS) and neuromyelitis optica spectrum disorder (NMOSD) are the two major inflammatory demyelinating diseases of the central nervous system[1,2]. Clinically distinguishing the two diseases is critical, because their prognoses and treatments differ ${ }^{2}$, and some MS treatments can exacerbate NMOSD[3,4]. Despite the existence of diagnostic criteria[5-7], the differential diagnosis of the two diseases can be difficult[8], especially at clinical onset. It is crucial to identify new effective biomarkers for quantifying the pathological alterations and accurately differentiating the two diseases, ideally biomarkers obtainable from routine clinical MRI data.

The principal MRI findings in both MS and NMOSD are spinal cord lesions, which are assessed visually, and described qualitatively based on the clinical imaging settings[2,9]. The lesion characteristics cannot, however, be evaluated quantitatively by visual inspection. Quantitative evaluation requires advanced analysis techniques. Radiomics is the process of converting medical images into high-dimensional, mineable data via high-throughput extraction of quantitative features, followed by subsequent data analysis for decision support[10,11]. Radiomic features have great potential to provide valuable information for clarifying pathophysiology, assisting in differential diagnosis, and guiding personalized therapy in MS and NMOSD. A nomogram uses a set of discriminative features derived from a regression model, and assigns each feature a weight that represents its value for clinical prediction ${ }^{12}$. 
This study aims to investigate the radiomic features of spinal cord lesions in MS and NMOSD, and to develop and validate a nomogram that incorporates the radiomic signature and other clinical variables, for individualized differential diagnosis of the two diseases. 


\section{Materials and Methods}

\section{Standard protocol approvals, registrations, and patient consents}

The institutional review board of *BLINDED*, approved the study, and written informed consent was obtained from each participant prior to participation.

\section{Participants}

A total of 189 patients with spinal cord lesions, including 95 patients with MS and 94 with NMOSD, were recruited from *BLINDED*. For the primary cohort, we retrospectively enrolled 67 MS and 68 NMOSD patients, from January 2015 to June 2016. A validation cohort was collected prospectively, including 28 consecutive patients with MS and 26 with NMOSD, from July 2016 to December 2016. The inclusion criteria for this study were: (1) a confirmed diagnosis of either NMOSD, according to the standard diagnosis criteria[6], or relapsing remitting MS (RRMS), according to the 2010 McDonald criteria[13]; (2) spinal cord lesions visible on T2 images; (3) being in remission (relapse-free for at least 4 weeks) and without treatment by disease-modifying medications within 4 weeks before the MRI scans, to exclude the confounding effects of edema or medication on the MRI measurements; (4) To exclude the possible diagnostic confounders of AQP4 negative NMO patients, all included patients with NMOSD were anti- AQP4 antibody positive. The exclusion criteria included: (1) a history of spinal cord injury or clinically significant neurologic 
disease other than MS or NMOSD; (2) image artifacts or incomplete clinical information. The principal demographic and clinical characteristics of the patients are shown in Table 1.

\section{MRI Acquisition}

All spinal cord MRI scans were performed using a 3.0 Tesla MR system (Siemens Magnetom Trio Tim system, Germany). Whole spinal cord (cervical, thoracic and lumbar) imaging included 3-mm-thick sagittal sections and 4-mm-thick axial sections using turbo spin-echo T2-weighted sequences (TR/TE: 3000/130 ms, in-plane resolution $1.0 \mathrm{~mm}^{2}$, field of view $=320 \times 260 \mathrm{~mm}^{2}$ ). Hyperintense cord lesions were marked as regions of interest (ROIs) on sagittal T2-weighted images by an experienced neuroradiologist $(* * *)$ using MRIcro software (http://www.mccauslandcenter.sc.edu/mricro/mricro/mricro.html).

\section{Radiomic methods}

In our study, we applied the emerging technique of radiomics to discriminate MS from NMOSD (figure 1). The process included mainly the following steps:(A) Feature extraction: we described the ROIs including the spinal cord lesions by extracting four sets of radiomic features[14]: (1) shape and size features, (2) gray scale intensity features, (3) textural features, and (4) wavelet features. Shape and size features represented the basic geometric features of the ROIs, such as shape, area, volume, 
compactness, etc. Gray scale intensity features were based on the differences of signal intensity histogram and distribution within the ROIs. Textural features encoded the relationships between nearby voxels within ROIs. Wavelet features were derived from a transformation of the grayscale intensity and texture features. Further information about the specific radiomic features is shown in the supplementary materials (figure S1-3). (B) Feature selection: to determine representative features for generalizing and optimizing the model, we used the least absolute shrinkage and selection operator (LASSO) method to select features for building a logistic regression model.

\section{Radiomic nomogram construction and validation}

We built a predictive model for differentiating MS from NMOSD using the radiomic features combined with several clinical variables, and a receiver operating characteristic (ROC) curve was plotted to quantify the performance of the model. An individual radiomic nomogram was developed using multivariable logistic regression based on discriminative predictors for the primary cohort. To compare the performance of radiomic model and clinically routine methods, we also built two other models for differentiating NMOSD and MS: (1) longitudinal extensive transverse myelitis (LETM) and (2) only radiomic features.

To quantify the discrimination of the radiomic nomogram, we generated a calibration plot for it, and also calculated Harrell's significant concordance index (C-index). 
Bootstrapping validation, with 1000 bootstrap resamples, was used to obtain the C-index.

\section{Statistical analysis}

We performed statistical analysis using Matlab 2015b (MathWorks, Natick, MA, USA) and $\mathrm{R}$ software, version 3.3.3 (http://www.R-project.org). We used SPM12 from Matlab 2015b to analyze the original MRI scans, for feature extraction and feature selection. The R packages "glmnet," "rms," and "Hmisc" were used for LASSO binary logistic regression and nomogram construction. A radiomic nomogram was constructed based on the results of the multivariable analysis, using the package "rms". We calculated the C-index, to measure the performance of the nomogram, using the package "Hmisc". Discrete data encoding the sexes of the patients were analyzed using the chi-square test. Two-sided two-sample t-tests or Wilcoxon rank sum tests were used to assess between-group differences for continuous demographic or clinical data, depending on whether they were normally distributed (Lilliefors test). A result was considered statistically significant if the $P$ value was less than 0.05 . 


\section{Results}

\section{Clinical data analysis}

The demographic and clinical characteristics of the patients with MS and NMOSD are summarized in table 1 . The NMOSD patients showed a greater female predominance, and higher expanded disability status scale (EDSS) values, than the MS patients, in both the primary and validation cohorts. There were no significant differences between the primary and validation cohorts in terms of clinical variables $(P>0.05)$.

\section{Radiomic features}

To differentiate MS and NMOSD, we extracted 485 radiomic features from the ROIs, plus 6 clinical and routine MRI measurements: sex, age, disease duration, number of relapses, EDSS score, spinal cord lesion length, and the number of cord lesions. The process is shown in figure $\mathbf{S 4}$. After feature selection, the initial 485 radiomic features were reduced to 9 features, and the 6 potential clinical and routine MRI characteristics were reduced to 3 variables, which were used to develop the LASSO logistic regression model. The features used were WLLH_GLCM_cluster_tendency

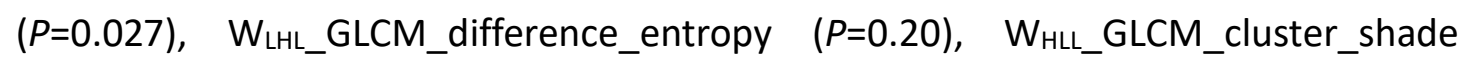
$(P=0.098), \quad$ GLRLM_SRE $\quad\left(P=3.5 \times 10^{-5}\right), \quad$ WLHLGLRLM_LRE $\quad(P=0.077)$,

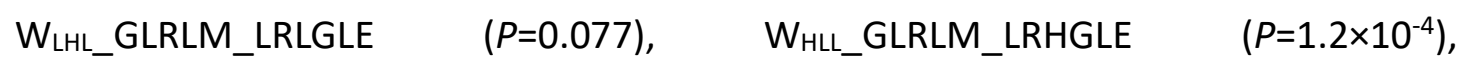

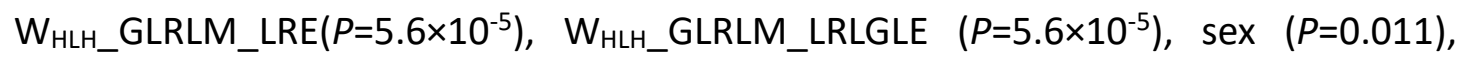
length $\left(P=4.6 \times 10^{-5}\right)$, and EDSS score $(P=0.05)$. The definitions of these features are shown in the supplementary materials (table S1, table S2). 
We depicted receiver operating characteristic (ROC) curves to assess the performance of different models. Firstly, we developed clinical model by routine clinical method (LETM model) to discriminate the NMOSD and MS, and the areas under the ROC curves (AUC) were 0.623 and 0.560 for the primary and validation cohorts, respectively (figure 2a). The model with only radiomic features demonstrated the AUC were 0.836 and 0.731 in primary and validation cohorts (figure 2b). Particularly, when we constructed the model with the radiomic features in combination with clinical variables, the AUC were 0.8808 and 0.7115 in the primary and validation cohorts (figure 2c). We found that the accuracy of model by radiomic features combining with clinical variables were $26 \%$ (in the primary cohort) and $15 \%$ (in validation cohort) higher than the routine clinical method (LETM model). We calculated a radiomic score to represent the value predicted by the LASSO regression model for each patient. The distribution of scores was shown in the supplementary materials (figure S5-6). The mean values of the radiomic score for MS and NMOSD, respectively, were 0.335 and -0.272 in the primary cohort, and 1.553 and -0.786 in the validation cohort.

In consideration of the fact that MS and NMOSD occur more frequently in female patients, we also investigated the discriminative performance of the radiomic features in the female cohort. The AUCs were 0.898 and 0.6684 for the female primary and validation cohorts, respectively (figure S7).

\section{Radiomic nomogram and validation}

An individualized prediction model for discriminating MS and NMO was developed 
using the multivariable logistic regression analysis, and represented by a nomogram (figure 3). The C-index for the nomogram was $0.8902(95 \% \mathrm{Cl}, 0.851$ to 0.932$)$. Calibration plots were used to correct the predictions of the radiomic nomogram for the primary cohort, to satisfy the Hosmer-Lemeshow test. We calculated the C-index and 1000 bootstrap resamples for the corrected version of the nomogram. For the primary cohort, the corrected C-index was 0.870 via bootstrapping validation. For the validation cohort, the C-index of the calibrated version of the radiomic nomogram was $0.804(95 \% \mathrm{Cl}, 0.690$ to 0.917$)$ and the corrected C-index was 0.782 via bootstrapping validation (figure 4). 


\section{Discussion}

In this study, we identified differences between the radiomic features of spinal cord lesions in MS and NMOSD, and developed and validated a nomogram combining radiomic features with clinical variables, to differentiate the two diseases.

Spinal cord lesions in MS and NMOSD are commonly observed clinically[9,15-16], but previous studies focused on visual assessment of properties of the lesions, such as the lesion length, lesion distribution, or lesion signal strength[17]. Complex patterns of pathology in lesions, which are commonly encountered in medical images, are difficult to interpret, and require advanced analysis techniques. Radiomics uses high-throughput advanced quantitative features to objectively and quantitatively describe the characteristics of lesions. These features, termed radiomic features, can be extracted from medical images using mathematical algorithms, with the goal of discovering lesion characteristics that may not be perceptible by the naked eye[10-11,14,18]. Thus, radiomics has great potential to capture important information for differential diagnosis and personalized therapy. The main radiomic features differentiating the two diseases (MS vs NMOSD) are measures of the heterogeneity of the lesion signal, such as WLL_GLCM_cluster_tendency and

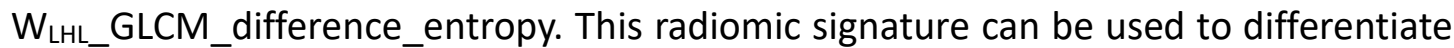
MS from NMOSD based on significantly different radiomic scores. A previous MRI-pathological study showed that greater MRI radiomic heterogeneity (i.e., stronger texture features) is associated with more severe pathological damage (more 
severe demyelination and greater axonal damage)[19]. Our radiomics results identified more severe pathological damage in NMOSD than in MS, which is consistent with pathological studies showing more severe demyelination and greater axonal loss in NMOSD than in MS, and with the observation that NMOSD lesions can show necrotic and cystic changes with extensive tissue destruction[20-21]. These features can be captured and quantified by radiomics, and may help to understand the pathophysiology of the disease. Furthermore, the radiomic model is superior (around 20\% increase in accuracy) than the clinically routine method which based on whether LETM present, highlighting its clinical importance.

A nomogram permits calculation of the cumulative effect of multiple differentiating factors[22]. By weighting the influence of each factor, the nomogram provides an appreciation of the relative magnitude of influence of each factor on the differential diagnosis. The advantage of a nomogram over an adjusted regression model is that, while the latter returns estimates of the average effects across a population, a nomogram permits individualized predictions[12]. A nomogram based on clinical and MRI measurement has been used to predict the clinical conversion of the clinically isolated syndrome[23], however, radiomic features were not included in the model. The radiomic features of cord lesions dominated the nomogram in terms of relative contribution to total points and differential diagnosis between the two diseases.

Spinal cord lesion length, sex, and EDSS score were also useful as factors in the nomogram, to help differentiate MS from NMOSD. This finding is consistent with our clinical observations and previous publications that patients with NMOSD showed 
greater lesion length, greater female predominance, and higher EDSS scores than patients with MS[2,16]. By combining these clinical and routine MRI features with radiomic signatures, the model can differentiate the two diseases accurately, highlighting the importance of comprehensive consideration of clinical and imaging features.

To avoid over-fitting or bias, we performed a robust statistical analysis. Representative radiomic features ( 9 features) were selected based on feature stability and prognostic performance in the cohort, and validated in an independent validation cohort. Internal and external validations of the radiomics nomogram were performed in the current study, and good calibration was observed, implying the robustness of the method and its potential clinical applications. To exclude the influence of an effect of sex[24-25], further validation was also performed in the female cohort (since female patients were more common in both MS and NMOSD). The radiomic nomogram showed equally good performance in distinguishing the two diseases as for the full patient cohort.

Several limitations apply to this work. First, this was a preliminary cross-sectional study using only spinal cord MR images. We did not evaluate the radiomic features of brain lesions, optic nerve lesions, or normal-appearing tissues. A longitudinal study with multimodal images, including the brain, spinal cord, and optic nerve, is warranted to investigate radiomic characteristics in other tissues, and dynamic changes in radiomic features, in MS and NMOSD. Second, it is unclear which pathological mechanisms are responsible for the radiomic heterogeneity[26], and 
how tissue repair may modify these features. Further studies are needed to confirm our findings and resolve these uncertainties. Finally, our study was a single center study using MR images from one MRI scanner. Further study using data from diverse scanners in a multicenter setting is required to validate our current findings and confirm their generalizability.

\section{Conclusion}

A validated nomogram that incorporates the radiomics signature combining with spinal cord lesions, cord lesion length, patient sex, and EDSS can well differential MS and NMOSD based on routine MRI data. 


\section{References}

1. Noseworthy JH, Lucchinetti C, Rodriguez M, Weinshenker BG.(2000) Multiple sclerosis. N Engl J Med 343:938-952.

2. Wingerchuk DM, Lennon VA, Lucchinetti CF, Pittock SJ, Weinshenker BG.(2007) The spectrum of neuromyelitis optica. Lancet Neurol 6:805-815.

3. Palace J, Leite MI, Nairne A, Vincent A.(2010) Interferon Beta treatment in neuromyelitis optica: increase in relapses and aquaporin 4 antibody titers. Arch Neurol 67:1016-1017.

4. Shimizu J, Hatanaka Y, Hasegawa M, et al.(2010) IFNbeta-1b may severely exacerbate Japanese optic-spinal MS in neuromyelitis optica spectrum. Neurology 75:1423-1427.

5. Thompson AJ, Banwell BL, Barkhof $F$, et al.(2017) Diagnosis of multiple sclerosis: 2017 revisions of the McDonald criteria. Lancet Neurol.

6. Wingerchuk DM, Banwell B, Bennett JL, et al.(2015) International consensus diagnostic criteria for neuromyelitis optica spectrum disorders. Neurology 85:177-189.

7. Min JH, Kim BJ, Lee KH. (2012) Development of extensive brain lesions following fingolimod (FTY720) treatment in a patient with neuromyelitis optica spectrum disorder. Mult Scler 18:113-115.

8. Jarius S, Ruprecht K, Wildemann B, et al. (2012) Contrasting disease patterns in seropositive and seronegative neuromyelitis optica: A multicentre study of 175 patients. J Neuroinflammation 9:14.

9. Gass A, Rocca MA, Agosta F, et al. (2015) MRI monitoring of pathological changes in the spinal cord in patients with multiple sclerosis. Lancet Neurol 14:443-454.

10. Lambin P, Rios-Velazquez E, Leijenaar R, et al. (2012) Radiomics: extracting more information from medical images using advanced feature analysis. Eur J Cancer 48:441-446.

11. Gillies RJ, Kinahan PE, Hricak H. (2016) Radiomics: Images Are More than Pictures, They Are Data. Radiology 278:563-577.

12. Iasonos A, Schrag D, Raj GV, Panageas KS. (2008) How to build and interpret a nomogram for cancer prognosis. J Clin Oncol 26:1364-1370.

13. Polman CH, Reingold SC, Banwell B, et al. (2011) Diagnostic criteria for multiple sclerosis: 2010 revisions to the McDonald criteria. Ann Neurol 69:292-302.

14. Aerts HJ, Velazquez ER, Leijenaar RT, et al. (2014) Decoding tumour phenotype by noninvasive imaging using a quantitative radiomics approach. Nat Commun 5:4006.

15. Lukas C, Knol DL, Sombekke MH, et al. (2015) Cervical spinal cord volume loss is related to clinical disability progression in multiple sclerosis. J Neurol Neurosurg Psychiatry 86:410-418. 
16. Liu Y, Wang J, Daams M, et al. (2015) Differential patterns of spinal cord and brain atrophy in NMO and MS. Neurology 84:1465-1472.

17. Fan M, Fu Y, Su L, et al. (2017) Comparison of brain and spinal cord magnetic resonance imaging features in neuromyelitis optica spectrum disorders patients with or without aquaporin-4 antibody. Mult Scler Relat Disord 13:58-66.

18. Kumar V, Gu Y, Basu S, et al. (2012) Radiomics: the process and the challenges. Magn Reson Imaging 30:1234-1248.

19. Zhang Y, Moore GR, Laule C, et al. (2013) Pathological correlates of magnetic resonance imaging texture heterogeneity in multiple sclerosis. Ann Neurol 74:91-99.

20. Kawachi I, Lassmann H. (2017) Neurodegeneration in multiple sclerosis and neuromyelitis optica. J Neurol Neurosurg Psychiatry 88:137-145.

21. Filippi M, Rocca MA, Barkhof $F$, et al. (2012) Association between pathological and MRI findings in multiple sclerosis. Lancet Neurol 11:349-360.

22. Wang Y, Li J, Xia Y, et al. (2013) Prognostic nomogram for intrahepatic cholangiocarcinoma after partial hepatectomy. J Clin Oncol 31:1188-1195.

23. Spelman T, Meyniel C, Rojas JI, et al. (2017) Quantifying risk of early relapse in patients with first demyelinating events: Prediction in clinical practice. Mult Scler 23:1346-1357.

24. Schoonheim MM, Vigeveno RM, Rueda Lopes FC, et al. (2014) Sex-specific extent and severity of white matter damage in multiple sclerosis: implications for cognitive decline. Hum Brain Mapp 35:2348-2358.

25. Borisow N, Kleiter I, Gahlen A, et al. (2017) Influence of female sex and fertile age on neuromyelitis optica spectrum disorders. Mult Scler 23:1092-1103.

26. Yip SS, Aerts HJ. (2016) Applications and limitations of radiomics. Phys Med Biol 61:R150-166. 


\section{Table and figure legends}

Table 1. Characteristics of patients in the primary and validation cohorts

Data are presented as mean \pm standard or median, depending on normality (Lilliefors test).

EDSS, Expanded Disability Status Scale; F, female; HC, healthy control; M, male; MS, multiple sclerosis; NMOSD, neuromyelitis optica spectrum disorder a $P$ values obtained using two-sample two-tailed t-tests.

${ }^{b} P$ values obtained using Pearson chi-square test.

${ }^{c} P$ values obtained using two-tailed Wilcoxon rank sum tests.

The radiomic score measures the strength of prediction for each patient. 
Figure 1. Radiomic procedure. (a) Original magnetic resonance images of patients. (b) Contours of lesions delineated by an experienced radiologist. (c) Extraction of features from the segmented regions of interest (ROIs), such as shape, intensity, texture and wavelet features. (d) Predictive analysis using least absolute shrinkage and selection operator (LASSO) regression model.

Figure 2. Receiver operating characteristic (ROC) curve analysis. Each model was constructed in primary cohort with 135 patients, and validated with 54 patients in validation cohort to test the model. (a) ROC curve analysis for the model constructed by the presence or absence of longitudinal extensive transverse myelitis (LETM). (b) ROC curves for the model constructed by radiomic feature. (c) ROC curve analysis for the model constructed by radiomic features and clinical variables.

Figure 3. Nomogram with radiomic and clinical variables. Using this tool, it is possible to generate a quantitative prediction for each patient by adding up the total number of points.

Figure 4. Calibration of the radiomic nomogram. (a) Calibration plot of the radiomic nomogram for the primary cohort of 135 patients. The C-index was $0.8902(95 \% \mathrm{Cl}$, 0.851 to 0.932 ). The corrected C-index was 0.870 via bootstrapping validation. (b) Calibration plot of the radiomic nomogram for the validation cohort of 54 patients. The C-index was $0.804(95 \% \mathrm{Cl}, 0.690$ to 0.917$)$. The corrected C-index was 0.782 via bootstrapping validation. We generated the calibration plot for the primary cohort to test discrimination of model prediction ability for MS and NMOSD. The X-axis 
represents the nomogram predicted probability; the Y-axis represents the actual probability. The blue diagonal dotted line represents an ideal prediction by an optimal model. The red diagonal dotted line represents the prediction by the nomogram. The black solid line represents the performance on multiple sets of bootstrap samples. 